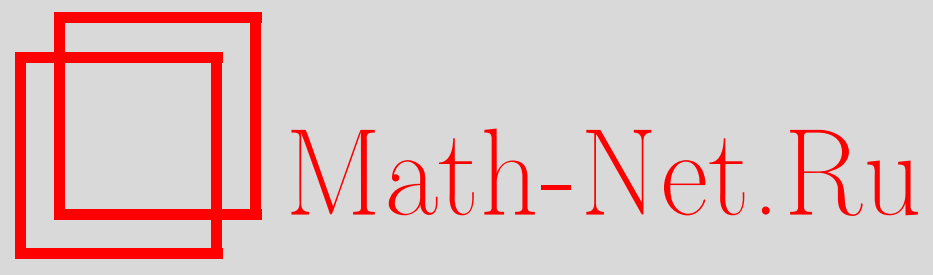

С. П. Горшков, О сложности задачи нахождения числа решений систем булевых уравнений, Дискрет. матем., 1996, том 8, выпуск 1, 72-85

DOI: https://doi.org/10.4213/dm509

Использование Общероссийского математического портала Math-Net.Ru подразумевает, что вы прочитали и согласны с пользовательским соглашением http: //www . mathnet.ru/rus/agreement

Параметры загрузки:

IP : 18.208 .226 .222

26 апреля 2023 г., $15: 27: 21$ 


\title{
О сложности задачи нахождения числа решений систем булевых уравнений
}

\author{
(c) 1996 г. С. П. Горшков
}

В работе рассматриваются классы систем булевых уравнений вида

$$
f_{s_{i}}\left(x_{s_{i 1}}, \ldots, x_{s_{i k_{i}}}\right)=1, \quad i=1, \ldots, m,
$$

где $m \in\{1,2, \ldots\}, x_{s_{i j}} \in\left\{x_{1}, x_{2}, \ldots\right\}, j=1, \ldots, k_{i}, i=1, \ldots, m$, функции $f_{s_{i}}$ выбираются из некоторого набора булевых функций

$$
F=\left\{f_{j}\left(x_{1}, \ldots, x_{k_{j}} \mid j \in J\right\} .\right.
$$

Задача определения числа решений систем уравнений такого класса обозначена enu $\left([\boldsymbol{F}]_{\mathrm{NC}}\right)$. Множество всех булевых функций, представимых конъюнкцией аффинных функций, обозначено $A$. Показано, что если $F \subseteq A$, то задача enu $\left([F]_{\mathrm{NC}}\right)$ является полиномиальной, а если $F \nsubseteq A$, то задача еnu $\left([F]_{\mathrm{NC}}\right)$ является \#Р-полной (труднорешаемой).

\section{1. Постановка задачи, комментарии}

$K$ проблеме решения систем булевых уравнений сводится ряд задач дискретной математики (см., например, $[1,2])$. Важным направлением исследования этой проблемы является изучение такой характеристики систем уравнений, как число решений. В настоящей работе рассматриваются так называемые системы булевых уравнений без ограничений на выбор неизвестных, которые, в частности, возникают при анализе булевых формул из известной задачи "обобщенная выполнимость" [3-5].

Введем необходимые понятия. Под классом систем булевых уравнений далее понимаем бесконечное множество систем булевых уравнений определенного вида (например, множество систем булевых линейных уравнений), в которых функции левых частей записаны некоторыми булевыми формулами. При необходимости будем указывать, какими именно булевыми формулами (например, многочленами Жегалкина, дизъюнктивными нормальными формами) записаны функции левых частей систем уравнений. Пусть $\mathbf{N}$ - множество натуральных чисел; $B_{n}-n$-мерное пространство двоичных векторов; $R$ - некоторый класс систем уравнений; $S\left(x_{q_{1}}, \ldots, x_{q_{n}}\right)$ - некоторая система из $R$, зависящая от неизвестных $x_{q_{1}}, \ldots, x_{q_{n}}$ (считаем, что система $S$ зависит от неизвестного $x_{q_{i}}$, если это неизвестное содержится в записи системы $S$ ).

Множество всех решений системы $S\left(x_{q_{1}}, \ldots, x_{q_{n}}\right)$ обозначим $\operatorname{sol}\left(S\left(x_{q_{1}}, \ldots, x_{q_{n}}\right)\right)$.

Сформулируем некоторые массовые задачи [3], связанные с решением систем уравнений класса $R$. 
(1) Задача распознавания совместности систем класса $R$.

Условие. Задана система $S \in R$.

Bonрос. Верно ли, что $|\operatorname{sol}(S)|>0$ ?

(2) Задача определения числа решений систем класса $R$.

Условие. Задана система $S \in R$.

Bonpoc. Какова мощность множества $\operatorname{sol}(S)$ ?

Задачи 1,2 обозначим, соответственно, $\operatorname{sat}(R), \operatorname{enu}(R)$. Очевидно, что задача $\operatorname{sat}(R)$ не сложнее задачи еnu $(R)$. Известные результаты о сложности задач $\operatorname{sat}(R)$ и enu $(R)$ для ряда классов систем уравнений $R$ приведем ниже.

Задачи вида еnu $(R)$ являются частным случаем задач перечисления, на которых остановимся подробнее (полную постановку вопроса см. в разделе 5.4 работы [6] или в разделе 7.3 монографии [3]). Пусть в массовой задаче $W$ каждая индивидуальная задача $w \in W$ имеет ассоциированное с ней множество peшений $\operatorname{sol}(w)$. Задача перечисления, соответствующая задаче $W$, формулируется так: задана $w \in W$, какова мощность множества $\operatorname{sol}(w)$ ?

Считающей машиной Тьюринга называется недетерминированная машина Тьюринга с вспомогательным устройством, в котором подсчитывается число ветвей вычислений, приводящих к решению. Функция сложности $\varphi(n)$ есть длина самого продолжительного пути вычисления по всем входам размера $n$.

Обозначим через \#Р класс задач перечисления, которые могут быть решены за полиномиальное время на подходящей считающей машине Тьюринга, а через $\mathrm{P}^{\prime}$ - класс задач перечисления, которые могут быть решены за полиномиальное время (с полиномиальной сложностью) на подходящей детерминированной машине Тьюринга. Задачи класса $\mathrm{P}^{\prime}$ называются полиномиальными.

Пример. Задача определения числа решений систем булевых линейных уравнений

$$
\alpha_{i 1} x_{1} \oplus \ldots \oplus \alpha_{i n} x_{n}=\alpha_{i 0}, \quad i=1, \ldots, m
$$

является полиномиальной. Соответствующий полиномиальный алгоритм просто строится на основе следующих фактов:

(а) задачи вычисления ранга матрицы и определения совместности систем булевых линейных уравнений являются полиномиальными;

(b) если система булевых линейных уравнений совместна, то она имеет $2^{n-r}$ решений, где

$$
r=\operatorname{rank}\left(\begin{array}{ccc}
\alpha_{i 1} & \ldots & \alpha_{i n} \\
& \ldots & \\
\alpha_{m 1} & \ldots & \alpha_{m n}
\end{array}\right)
$$

Говорят, что задача перечисления $W_{2}$ сводится по Тьюрингу к задаче перечисления $W_{1}$, и записывают $W_{2} \propto_{\mathrm{T}} W_{1}$, если в случае существования полиномиального алгоритма решения $W_{1}$ может быть построен полиномиальный алгоритм решения $W_{2}$. 
Задача $W_{1}$ называется \#Р-полной, если для любой задачи $W_{2} \in \# \mathrm{P}$ имеет место сводимость $W_{2} \propto_{\mathrm{T}} W_{1}$.

$\mathrm{B}$ настоящее время гипотеза $\mathrm{P}^{\prime} \neq \# \mathrm{P}$, так же как и гипотеза $\mathrm{P} \neq \mathrm{NP}$, ни доказана, ни опровергнута. \#Р-полные задачи являются, по-видимому, более сложными, чем NP-полные задачи; даже в случае $\mathrm{P}=\mathrm{NP}$ совершенно неясно, решаются ли \#Р-полные задачи за полиномиальное время. \#Р-полные задачи относятся к разряду труднорешаемых задач [3].

Классы \#Р-задач и \#Р-полных задач были введены в работе [7] (аналогичное предложение было сделано в [8]). В работе [9] продолжено изучение этой тематики, доказана \#Р-полнота многих задач перечисления, в том числе задачи определения числа решений систем Булевых уравнений вида

$$
x_{s_{i 1}} \vee x_{s_{i 2}}=1, \quad i=1, \ldots, m,
$$

где $m \in \mathbf{N}, x_{s_{i j}} \in\left\{x_{1}, x_{2}, \ldots\right\}, i=1, \ldots, m, j=1,2$. Пусть $R-$ произвольный класс систем булевых уравнений. Используя тот факт, что с полиномиальной сложностью можно вычислить значение всякой булевой формулы на любом фиксированном наборе значений переменных [10, с. 419], нетрудно построить считающую машину Тьюринга, решающую задачу еnu $(R)$ за полиномиальное время. Поэтому enu $(R) \in \# \mathrm{P}$ и, если для некоторой \#Р-полной задачи enu $\left(R^{\prime}\right)$, где $R^{\prime}$ - некоторый класс систем уравнений, удается показать сводимость епu $\left(R^{\prime}\right) \propto_{\mathrm{T}}$ епu $(R)$, то задача еnu $(R)$ \#Р-полна.

Пусть $\mathbf{N}_{0}=\{\mathbf{N} \cup 0\}$. Для доказательства сводимости enu $\left(R^{\prime}\right) \propto_{\mathrm{T}} \operatorname{enu}(R)$ будем строить вычисляемые за полиномиальное время функцию $\psi_{1}: \mathbf{N}_{0} \rightarrow \mathbf{N}_{0}$ и функцию $\psi_{2}$, сопоставляющую каждой системе $S^{\prime} \in R^{\prime}$ систему $\psi_{2}\left(S^{\prime}\right) \in R$, обладающие свойством $\left|\operatorname{sol}\left(S^{\prime}\right)\right|=\psi_{1}\left(\left|\operatorname{sol}\left(\psi_{2}\left(S^{\prime}\right)\right)\right|\right)$. Такая сводимость называется консервативной сводимостью (см. $[3$, с. 210]).

Определим классы систем булевых уравнений, которые будем рассматривать далее. Пусть $X=\left\{x_{j} \mid j \in \mathbf{N}\right\}, F=\left\{f_{j}\left(x_{1}, \ldots, x_{k_{j}}\right) \mid j \in J\right\}$ - конечный или бесконечный набор булевых функций. Считаем, что функции набора $F$ записаны булевыми формулами (в дальнейшем для краткости слово булевы иногда будем опускать).

Символом $[F]_{\mathrm{NC}}$ обозначим класс всевозможных систем уравнений, каждое уравнение которых имеет следующую структуру: правая часть равна 1 , левая часть есть функция из набора $F$, переменные выбираются из множества $X$; других ограничений на системы класса $[F]_{\mathrm{NC}}$ не накладывается. Так, если $F=\left\{f_{j}\left(x_{1}, \ldots, x_{k}\right) \mid j=1, \ldots, d\right\}-$ конечный набор булевых функций, то систе-

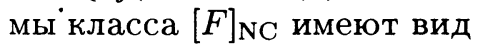

$$
f_{s_{i}}\left(x_{s_{i 1}}, \ldots, x_{s_{i k}}\right)=1, \quad i=1, \ldots, m,
$$

где $m \in \mathbf{N}, f_{s_{i}} \in F, x_{s_{i j}} \in X, i=1, \ldots, m, j=1, \ldots, k$.

Такие системы называются системами булевых уравнений без ограничений на выбор неизвестных. Системы класса $[F]_{\mathrm{NC}}$ являются аналогами S-формул из работы [4]. Заметим, что в виде $[F]_{N C}$ могут быть записаны многие классы систем уравнений. Так, например, если

$$
F=\left\{x_{1} \oplus \ldots \oplus x_{k} \oplus \alpha \mid k \in \mathbf{N}, \alpha \in\{0,1\}\right\},
$$

то $[F]_{\mathrm{NC}}-$ множество всех систем линейных уравнений; если

$$
F=\left\{x_{1}^{\alpha_{1}} \vee \ldots \vee x_{k}^{\alpha_{k}} \mid \alpha_{i} \in\{0,1\}, i=1, \ldots, k\right\},
$$


то множество $[F]_{\mathrm{NC}}$ эквивалентно классу всех систем, каждое уравнение которых зависит не более чем от $k$ неизвестных.

Функция $f\left(x_{1}, \ldots, x_{k}\right)$ называется мультиаффинной [4], если существует представление функции $f$ в виде конъюнкции аффинных (степени нелинейности не выше первой) функций

$$
f=\bigwedge_{i=1}^{t}\left(\alpha_{i 1} x_{1} \oplus \ldots \oplus \alpha_{i k} x_{k} \oplus \alpha_{i 0}\right)
$$

где $\alpha_{i j} \in\{0,1\}, i=1, \ldots, t, j=0, \ldots, k$. Множество всех мультиаффинных функций обозначим $A$. Запись (4) будем называть приведенным представлением мультиаффинной функции $f$.

В разделе 2 настоящей работы, при некоторых ограничениях на запись функций бесконечных наборов $F \subseteq A$, доказана следующая теорема разделимости: если для набора булевых функций $F$ выполняется включение $F \subseteq A$, то задача enu $\left([F]_{\mathrm{NC}}\right)$ полиномиальна, в случае $F \nsubseteq A$ задача enu $\left([F]_{\mathrm{NC}}\right)$ является \#Р-полной.

Термин разделимость употребляется в том смысле, что любая задача вида enu $\left([F]_{\mathrm{NC}}\right)$ оказывается либо полиномиальной, либо \#Р-полной. Заметим, что для задач вида $\operatorname{sat}\left([F]_{\mathrm{NC}}\right)$ также имеет место разделимость на полиномиальные и NP-полные.

Согласно [4], функция $f\left(x_{1}, \ldots, x_{k}\right)$ называется 0-выполнимой, если $f(0, \ldots, 0)=1,1$-выполнимой, если $f(1, \ldots, 1)=1$, биюнктивной, если

$$
f=\bigwedge_{i=1}^{t}\left(x_{s_{i 1}}^{\alpha_{i 1}} \vee x_{s_{i 2}}^{\alpha_{i 2}}\right)
$$

слабо положительной, если

$$
f=\bigwedge_{i=1}^{t}\left(x_{s_{i 1}}^{\alpha_{i}} \vee x_{s_{i 2}} \vee \ldots \vee x_{s_{i k_{i}}}\right)
$$

слабо отрицательной, если

$$
f=\bigwedge_{i=1}^{t}\left(x_{s_{i 1}}^{\alpha_{i}} \vee \bar{x}_{s_{i 2}} \vee \ldots \vee \bar{x}_{s_{i k_{i}}}\right) .
$$

Множество всех функций этих классов обозначим соответственно 0-S, 1-S, Bi, $\mathrm{WP}, \mathrm{WN}$.

В [4] получен следующий результат. Если для конечного набора булевых функций $F$, не равных тождественно нулю, выполняется хотя бы одно из включений

$$
F \subset A, \quad F \subset \mathrm{Bi}, \quad F \subset \mathrm{WP}, \quad F \subset \mathrm{WN}, \quad F \subset 0-\mathrm{S}, \quad F \subset 1-\mathrm{S},
$$

то задача $\operatorname{sat}\left([F]_{\mathrm{NC}}\right)$ полиномиальна. Если не выполняется ни одно из включений $(5)$, то задача $\operatorname{sat}\left([F]_{N C}\right)$ является NP-полной. Заметим, что в [3] этот результат приведен с искажениями. 
Таким образом, сравнивая результаты настоящей работы и [4], можно констатировать, что только в случае $F \subseteq A$ задачи $\operatorname{sat}\left([F]_{\mathrm{NC}}\right)$ и enu( $\left.[F]_{\mathrm{NC}}\right)$ полиномиальны; если $F \nsubseteq A$, но выполняется хотя бы одно другое включение из $(5)$, задача $\operatorname{sat}\left([F]_{\mathrm{NC}}\right)$ полиномиальна, а задача enu $\left.[F]_{\mathrm{NC}}\right)$ \#P-полна. Если не выполняется ни одно из включений (5), то задача sat $\left.[F]_{\mathrm{NC}}\right)$ NP-полна, а задача enu $\left.[F]_{\text {NC }}\right)$ \#Р-полна.

Обозначим $F \oplus 1=\left\{f_{j} \oplus 1 \mid f_{j} \in F\right\}$. Системы класса $F \oplus 1$ можно рассматривать как системы класса $[F]_{\mathrm{NC}}$ с тем лишь отличием, что в правой части систем встречаются как единицы, так и нули (системы с произвольной правой частью). Легко показать, что если $\{F, F \oplus 1\} \subseteq A$, то все функции класса $F$ являются аффинными. Следовательно, в случае справедливости гипотезы $\mathrm{P}^{\prime} \neq \# \mathrm{P}$, задачи вида еnu $\left([F, F \oplus 1]_{\mathrm{NC}}\right)$ имеют полиномиальную сложность тогда и только тогда, когда $[F, F \oplus 1]_{\text {NC }}$ является подклассом класса систем линейных уравнений.

\section{2. Теорема разделимости}

Для дальнейшего изложения введем несколько обозначений. Если $f\left(x_{1}, \ldots, x_{k}\right)$ - функция, $\alpha=\left(\alpha_{1}, \ldots, \alpha_{k}\right) \in B_{k}, \beta=\left(\beta_{1}, \ldots, \beta_{k}\right) \in B_{k}$ - двоичные векторы, то полагаем

$$
\begin{gathered}
\alpha \oplus \beta=\left(\alpha_{1} \oplus \beta_{1}, \ldots, \alpha_{k} \oplus \beta_{k}\right), \quad f(\alpha)=f\left(\alpha_{1}, \ldots, \alpha_{k}\right), \\
0_{k}=(0, \ldots, 0) \in B_{k}, \quad 1_{k}=(1, \ldots, 1) \in B_{k} .
\end{gathered}
$$

Символом $\langle f\rangle_{\mathrm{C}}$ обозначим набор булевых функций

$$
\left\{f\left(y_{p_{1}}, \ldots, y_{p_{k}}\right) \mid y_{p_{i}} \in\left\{0,1, x_{1}, \ldots, x_{k}\right\}, i=1, \ldots, k\right\}
$$

(символ $y_{p_{i}}$ обозначает константу 0 или 1 или одну из переменных $x_{1}, \ldots, x_{k}$ );

$$
\langle f\rangle_{0}=\left\{f\left(z_{p_{1}}, \ldots, z_{p_{k}}\right) \mid z_{p_{i}} \in\left\{0, x_{1}, \ldots, x_{k}\right\}, i=1, \ldots, k\right\} .
$$

Классы систем уравнений $\left[\langle f\rangle_{\mathrm{C}}\right]_{\mathrm{NC}},\left[\langle f\rangle_{0}\right]_{\mathrm{NC}}$ обозначим соответственно $[f]_{\mathrm{C}},[f]_{0}$.

Если $\alpha=\left(\alpha_{1}, \ldots, \alpha_{k}\right) \in B_{k}, f\left(\alpha_{1}, \ldots, \alpha_{k}\right)=1$, то $\alpha$ назовем выполняющим вектором функции $f$. Множество всех выполняющих векторов функции $f$ обозначим $E_{f}$.

Теорема. Если все функиии набора $F$ являются мультиаффинжыми и в случае $|F|=\infty$ функции набора $F$ записаны или соответствующими приведенными представлениями, или многочленами Жегалкина, или дизбюнктивными нормальными формами, то задача епu $\left([F]_{\mathrm{NC}}\right)$ полиномиальна. Если в наборе $F$ найдется не мультиаффинная функция, то задача епu $\left([F]_{\mathrm{NC}}\right)$ \#P-полна.

Доказательство. Если набор функций $F \subset A$ конечен (при этом функции из $F$ первоначально могут быть записаны в любом виде), то со сложностью не более некоторой константы, зависящей от набора $F$, все функции из $F$ могут быть записаны приведенными представлениями.

Пусть $F \subseteq A$ и все функции набора $F$ записаны приведенными представлениями. Тогда любая система класса $[F]_{\mathrm{NC}}$ с полиномиальной сложностью сводится к соответствующей системе линейных уравнений вида (1), у которой множество 
решений совпадает с множеством решений исходной системы уравнений. Поскольку задача определения числа решений систем линейных уравнений является полиномиальной, то задача еnu $\left([F]_{\mathrm{NC}}\right)$ также полиномиальна.

Для доказательства существования полиномиального алгоритма решения задачи еnu $\left([F]_{\mathrm{NC}}\right)$, при условии, что $F \subseteq A$ и функции набора $F$ записаны или многочленами Жегалкина, или дизъюнктивными нормальными формами (ДНФ), достаточно показать, что существует полиномиальный алгоритм нахождения по многочлену Жегалкина или ДНФ приведенного представления функции. В этом случае полиномиальный алгоритм определения числа решений систем класса $[F]_{\mathrm{NC}}$ состоит из двух этапов:

(1) все функции левой части каждой решаемой системы $(3)$ из класса $[F]_{\mathrm{NC}}$ заменяем приведенным представлением функций,

(2) находим число решений полученной системы.

Пусть функция $f\left(x_{1}, \ldots, x_{k}\right) \in A$ представлена многочленом Жегалкина. Без ограничения общности можем считать, что $k \geqslant 1$. В случае $f \equiv 0$, приведенное представление функции имеет вид $f \equiv x_{1} \bar{x}_{1}$. Далее считаем $f \not \equiv 0$. Очевидно, что, если аффинная функция $\alpha_{1} x_{1} \oplus \ldots \oplus \alpha_{k} x_{k} \oplus \alpha_{0}$ является сомножителем в некотором приведенном представлении функции $f$, то

$$
\left(\alpha_{1} x_{1} \oplus \ldots \oplus \alpha_{k} x_{k} \oplus \alpha_{0}\right) f \equiv f .
$$

Используя соотношение (6), составим систему линейных уравнений (обозначим ее $L_{f}$ ) относительно неизвестных коэффициентов $\alpha_{0}, \ldots, \alpha_{k}$. Нетрудно видеть, что конъюнкция всех аффинных функций, соответствующих линейно независимым решениям линейной системы $L_{f}$, является приведенным представлением функции $f$.

Предположим теперь, что функция $f \not \equiv 0$ представлена дизъюнктивной нормальной формой, $E_{f}$ - множество выполняющих векторов функции $f$. Заметим, что поскольку $f$ - мультиаффинная функция, то $E_{f}$ есть множество решений некоторой системы линейных уравнений.

Так как $f$ записана в виде ДН $\Phi$, с полиномиальной сложностью находим некоторый вектор $\beta=\left(\beta_{1}, \ldots, \beta_{k}\right) \in E_{f}$. Множество $E_{f} \oplus \beta=\left\{\alpha \oplus \beta \mid \alpha \in E_{f}\right\}$ является множеством выполняющих векторов функции $f^{\prime}\left(y_{1}, \ldots, y_{k}\right)$, получаемой из $f$ заменой переменных

$$
x_{i}=y_{i}^{\left(\beta_{i} \oplus 1\right)}, \quad i=1, \ldots, k
$$

Кроме того, множество $E_{f} \oplus \beta$ есть некоторое линейное пространство векторов.

В функции $f$ проведем замену переменных (7). Пусть ДНФ функции $f^{\prime}$ принадлежит импликанта $y_{1}^{\delta_{1}} \ldots y_{t}^{\delta_{t}}$. Выпишем вектора длины $k:\left(\delta_{1}, \ldots, \delta_{t}\right)$, если $t=k ;\left(\delta_{1}, \ldots, \delta_{t}, 1,0, \ldots, 0\right), \ldots,\left(\delta_{1}, \ldots, \delta_{t}, 0, \ldots, 0,1\right),\left(\delta_{1}, \ldots, \delta_{t}, 0, \ldots, 0\right)$, если $t<k$ (здесь число элементов, дополняющих вектор до размера $t$ равно $k-t$ ). Проделаем эту процедуру для всех импликант ДН $\Phi$ функции $f^{\prime}$. Полученное множество векторов обозначим $T_{f^{\prime}}$. Ясно, что $T_{f^{\prime}} \subseteq E_{f} \oplus \beta$.

Базис пространства $E_{f} \oplus \beta$ может быть составлен из векторов множества $T_{f^{\prime}}$. Выберем этот базис и обозначим его $\gamma^{(1)}, \ldots, \gamma^{(q)}$. 
Далее подберем такую невырожденную замену переменных

$$
y_{i}=l_{i}\left(z_{1}, \ldots, z_{k}\right), \quad i=1, \ldots, k,
$$

при которой множество выполняющих векторов функции $f^{\prime \prime}\left(z_{1}, \ldots, z_{k}\right)=$ $f^{\prime}\left(l_{1}\left(z_{1}, \ldots, z_{k}\right), \ldots, l_{k}\left(z_{1}, \ldots, z_{k}\right)\right)$ имело бы базис $e^{(1)}, \ldots, e^{(q)}$, где в $e^{(i)}=$ $(0, \ldots, 0,1,0, \ldots, 0) \in B_{k}$ единица стоит на $i$-м месте, $i=1, \ldots, q$. При этом $f^{\prime \prime}\left(z_{1}, \ldots, z_{k}\right)=\bar{z}_{q+1} \ldots \bar{z}_{k}$. Проведя замены, обратные к (8) и (7), получим приведенное представление функции $f$.

Легко показывается, что указанные алгоритмы имеют полиномиальную сложность.

Предположим теперь, что $F \nsubseteq A$. Через $f\left(x_{1}, \ldots, x_{k}\right)$ обозначим некоторую не мультиаффинную функцию набора $F$. Покажем, что задача enu $\left([f]_{\mathrm{NC}}\right)$ является \#Р-полной, откуда будет следовать \#Р-полнота задачи еnu $\left([F]_{\mathrm{NC}}\right)$. Доказательство второй части теоремы разобьем на несколько лемм.

Как было отмечено выше, в [9] доказана \#Р-полнота задачи enu $\left(\left[x_{1} \vee x_{2}\right]_{\mathrm{NC}}\right)$ (см. (2)), откуда очевидно следует \#Р-полнота задачи enu([ $\left.\left.\bar{x}_{1} \vee \bar{x}_{2}\right]_{\mathrm{NC}}\right)$.

Лемма 1. Задача еnu $\left(\left[x_{1} \vee \bar{x}_{2}\right]_{\mathrm{NC}}\right)$ \#P-полна.

Доказательство. Покажем, что

$$
\operatorname{enu}\left(\left[x_{1} \vee x_{2}\right]_{\text {NC }}\right) \propto_{\mathrm{T}} \operatorname{enu}\left(\left[x_{1} \vee \bar{x}_{2}\right]_{\mathrm{NC}}\right)
$$

Сначала заметим, что существует полиномиальный алгоритм, который для всякой системы $S$, зависящей от $n$ неизвестных, может таким образом перенумеровать неизвестные, чтобы система $S$ зависела от неизвестных $x_{1}, \ldots, x_{n}$.

Рассмотрим некоторую систему $(2)$ из класса $\left[x_{1} \vee x_{2}\right]_{\mathrm{NC}}$, неизвестными которой являются $x_{1}, \ldots, x_{n}$. Системе $(2)$ сопоставим систему из класса $\left[x_{1} \vee \bar{x}_{2}\right]_{\mathrm{NC}}$

$$
\begin{array}{ll}
x_{i(n+1)+j} \vee \bar{x}_{s_{i 1}}=1, & i=1, \ldots, m, \\
x_{i(n+1)+j} \vee \bar{x}_{s_{i 2}}=1, & j=0, \ldots, n .
\end{array}
$$

Для всякого $h \in\{0, \ldots, m\}$ через $q_{h}$ обозначим число двоичных векторов $\left(\alpha_{1}, \ldots, \alpha_{n}\right)$ длины $n$, каждый из которых, при подстановке в систему (2) значений $x_{i}=\alpha_{i}, i=1, \ldots, n$, не удовлетворяет ровно $h$ уравнениям системы (2). Заметим, что при этом символом $q_{0}$ обозначено число решений системы (2).

Число решений $t$ системы (9) следующим образом выражается через величины $q_{h}, h=0, \ldots, m$ :

$$
t=\sum_{h=0}^{m} q_{h} 2^{h(n+1)} .
$$

Следовательно, $q_{0}$ есть остаток от деления числа $t$ на $2^{n+1}$. Дальнейшее доказательство сводимости по Тьюрингу еnu $\left(\left[x_{1} \vee x_{2}\right]_{\text {NC }}\right) \propto_{\mathrm{T}}$ enu $\left(\left[x_{1} \vee \bar{x}_{2}\right]_{\mathrm{NC}}\right)$ очевидно. Таким образом, задача enu $\left(\left[x_{1} \vee \bar{x}_{2}\right]_{\text {NC }}\right)$ \#Р-полна. Лемма доказана.

Замечание. Функция $x_{1} \vee \bar{x}_{2}$ является биюнктивной, слабо положительной и слабо отрицательной, в то время как функция $x_{1} \vee x_{2}$ является биюнктивной и слабо положительной, но не слабо отрицательной. 
Лемма 2. $Е с л и ~ f\left(x_{1}, \ldots, x_{k}\right) \notin A, f(0, \ldots, 0)=1$, то задача епu $\left([f]_{0}\right)$ \#Р-полна.

Доказательство. Поскольку функция $f$ не мультиаффинная, то по критерию мультиаффинности [4] найдутся три вектора $\alpha=\left(\alpha_{1}, \ldots, \alpha_{k}\right), \beta=\left(\beta_{1}, \ldots, \beta_{k}\right)$, $\gamma=\left(\gamma_{1}, \ldots, \gamma_{k}\right)$, такие, что

$$
f(\alpha)=f(\beta)=f(\gamma)=1, \quad f(\alpha \oplus \beta \oplus \gamma)=0 .
$$

Покажем, что в случае $f\left(0_{k}\right)=1$ можно выбрать три вектора, среди которых один нулевой, удовлетворяющие соотношениям (10). Если $f(\alpha \oplus \beta)=0$, то для векторов $0_{k}, \alpha, \beta$ выполняется (10). В случае $f(\alpha \oplus \beta)=1$ соотношения (10) справедливы для векторов $0_{k}, \alpha \oplus \beta, \gamma$. Далее будем полагать, что три вектора, для которых выполняется (10), есть $0_{k}, \beta, \gamma$.

В наборе $\langle f\rangle_{0}$ найдется такая функция $f^{\prime}\left(x_{1}, \ldots, x_{k}\right)=f\left(x_{p_{1}}, \ldots, x_{p_{k}}\right)$, отличающаяся от $f$ только перестановкой переменных, что

$$
\begin{aligned}
\left(\beta_{p_{1}}, \ldots, \beta_{p_{k}}\right) & =(0, \ldots, 0,0, \ldots, 0,1, \ldots, 1,1, \ldots, 1), \\
\left(\gamma_{p_{1}}, \ldots, \gamma_{p_{k}}\right) & =(0, \ldots, 0,1, \ldots, 1,0, \ldots, 0,1, \ldots, 1),
\end{aligned}
$$

где $r_{1}, r_{2}, r_{3}, r_{4}$ - длины последовательных частей вектора, состоящих сплошь из нулей и единиц, $r_{1}+\ldots+r_{4}=k$. Выберем из набора $\langle f\rangle_{0}$ функцию

$$
g\left(x_{1}, x_{2}, x_{3}\right)=f^{\prime}\left(0, \ldots, 0, x_{1}, \ldots, x_{1}, x_{2}, \ldots, x_{2}, x_{3}, \ldots, x_{3}\right),
$$

в которой аргументы начинаются $r_{1}$ нулей и продолжаются $r_{2}, r_{3}$ и $r_{4}$ переменными $x_{1}, x_{2}$ и $x_{3}$ соответственно. Очевидно, что $g(0,0,0)=g(0,1,1)=$ $g(1,0,1)=1, g(1,1,0)=0$. Возможные варианты функции $g\left(x_{1}, x_{2}, x_{3}\right)$ приведены в таблице:

\begin{tabular}{ccc|cccccccccccccccc}
$x_{1}$ & $x_{2}$ & $x_{3}$ & $g_{1}$ & $g_{2}$ & $g_{3}$ & $g_{4}$ & $g_{5}$ & $g_{6}$ & $g_{7}$ & $g_{8}$ & $g_{9}$ & $g_{10}$ & $g_{11}$ & $g_{12}$ & $g_{13}$ & $g_{14}$ & $g_{15}$ & $g_{16}$ \\
\hline 0 & 0 & 0 & 1 & 1 & 1 & 1 & 1 & 1 & 1 & 1 & 1 & 1 & 1 & 1 & 1 & 1 & 1 & 1 \\
0 & 0 & 1 & 0 & 0 & 0 & 0 & 0 & 0 & 0 & 0 & 1 & 1 & 1 & 1 & 1 & 1 & 1 & 1 \\
0 & 1 & 0 & 0 & 0 & 0 & 0 & 1 & 1 & 1 & 1 & 0 & 0 & 0 & 0 & 1 & 1 & 1 & 1 \\
0 & 1 & 1 & 1 & 1 & 1 & 1 & 1 & 1 & 1 & 1 & 1 & 1 & 1 & 1 & 1 & 1 & 1 & 1 \\
1 & 0 & 0 & 0 & 0 & 1 & 1 & 0 & 0 & 1 & 1 & 0 & 0 & 1 & 1 & 0 & 0 & 1 & 1 \\
1 & 0 & 1 & 1 & 1 & 1 & 1 & 1 & 1 & 1 & 1 & 1 & 1 & 1 & 1 & 1 & 1 & 1 & 1 \\
1 & 1 & 0 & 0 & 0 & 0 & 0 & 0 & 0 & 0 & 0 & 0 & 0 & 0 & 0 & 0 & 0 & 0 & 0 \\
1 & 1 & 1 & 0 & 1 & 0 & 1 & 0 & 1 & 0 & 1 & 0 & 1 & 0 & 1 & 0 & 1 & 0 & 1
\end{tabular}

Справедливы соотношения

$$
\begin{aligned}
& g_{2}\left(x_{1}, x_{2}, x_{1}\right)=x_{1} \vee \bar{x}_{2}, \quad g_{3}\left(x_{1}, 0, x_{2}\right)=x_{1} \vee \bar{x}_{2}, \\
& g_{4}\left(x_{1}, 0, x_{2}\right)=x_{1} \vee \bar{x}_{2}, \quad g_{5}\left(0, x_{1}, x_{2}\right)=x_{1} \vee \bar{x}_{2}, \\
& g_{6}\left(0, x_{1}, x_{2}\right)=x_{1} \vee \bar{x}_{2}, \quad g_{7}\left(0, x_{1}, x_{2}\right)=x_{1} \vee \bar{x}_{2} \text {, } \\
& g_{8}\left(0, x_{1}, x_{2}\right)=x_{1} \vee \bar{x}_{2}, \quad g_{9}\left(0, x_{1}, x_{2}\right)=\bar{x}_{1} \vee x_{2}, \\
& g_{10}\left(0, x_{1}, x_{2}\right)=\bar{x}_{1} \vee x_{2}, \quad g_{11}\left(0, x_{1}, x_{2}\right)=\bar{x}_{1} \vee x_{2} \text {, } \\
& g_{12}\left(0, x_{1}, x_{2}\right)=\bar{x}_{1} \vee x_{2}, \quad g_{13}\left(x_{1}, 0, x_{2}\right)=\bar{x}_{1} \vee x_{2} \text {, } \\
& g_{14}\left(x_{1}, 0, x_{2}\right)=\bar{x}_{1} \vee x_{2}, \quad g_{15}\left(x_{1}, x_{2}, 0\right)=\bar{x}_{1} \vee \bar{x}_{2} \text {, }
\end{aligned}
$$




$$
g_{16}\left(x_{1}, x_{2}, 0\right)=\bar{x}_{1} \vee \bar{x}_{2} .
$$

Следовательно, для задач епu $\left(\left[g_{i}\right]_{0}\right), i=2, \ldots, 16$, имеет место хотя бы одна из сводимостей еnu $\left(\left[x_{1} \vee \bar{x}_{2}\right]_{\mathrm{NC}}\right) \propto_{\mathrm{T}}$ enu $\left(\left[g_{i}\right]_{0}\right)$ или enu $\left(\left[\bar{x}_{1} \vee \bar{x}_{2}\right]_{\mathrm{NC}}\right) \propto_{\mathrm{T}}$ enu $\left(\left[g_{i}\right]_{0}\right)$. Используя лемму 1 и \#Р-полноту задачи еnu $\left(\left[\bar{x}_{1} \vee \bar{x}_{2}\right]_{\mathrm{NC}}\right)$, находим, что задачи enu $\left(\left[g_{i}\right]_{0}\right), i=2, \ldots, 16, \#$ Р-полны.

Рассмотрим функцию $g_{1}$. Покажем, что

$$
\operatorname{enu}\left(\left[\bar{x}_{1} \vee \bar{x}_{2}\right]_{\mathrm{NC}}\right) \propto_{\mathrm{T}} \operatorname{enu}\left(\left[g_{1}\right]_{0}\right) .
$$

Пусть система уравнений

$$
\bar{x}_{s_{i 1}} \vee \bar{x}_{s_{i 2}}=1, \quad i=1, \ldots, m,
$$

есть некоторая система из класса $\left[\bar{x}_{1} \vee \bar{x}_{2}\right]_{\text {NC }}$, зависящая от неизвестных $x_{1}, \ldots, x_{n}$. Системе (11) сопоставим систему из класса $\left[g_{1}\right]_{0}$

$$
g_{1}\left(x_{s_{i 1}}, x_{s_{i 2}}, x_{n+i}\right)=1, \quad i=1, \ldots, m .
$$

Заметим, что если вектор $\left(\alpha_{1}, \ldots, \alpha_{n}\right)$ - решение системы (11), то системе (12) удовлетворяет вектор $\left(\alpha_{1}, \ldots, \alpha_{n}, \alpha_{s_{11}} \vee \alpha_{s_{12}}, \ldots, \alpha_{s_{m 1}} \vee \alpha_{s_{m 2}}\right)$. С другой стороны, для всякого решения системы (12) $\left(\beta_{1}, \ldots, \beta_{n+m}\right) \in B_{n+m}$ выполняются соотношения

$$
\bar{\beta}_{s_{i 1}} \vee \bar{\beta}_{s_{i 2}}=1, \quad i=1, \ldots, m, \quad \beta_{n+i}=\beta_{s_{i 1}} \vee \beta_{s_{i 2} 2} \quad i=1, \ldots, m .
$$

На основе этих выкладок легко доказать, что число решений системы (11) равно числу решений системы (12). Поэтому еnu $\left(\left[\bar{x}_{1} \vee \bar{x}_{2}\right]_{\mathrm{NC}}\right) \propto_{\mathrm{T}}$ enu $\left(\left[g_{1}\right]_{0}\right)$ и задача enu $\left(\left[g_{1}\right]_{0}\right)$ \#Р-полна. Следовательно, при любых вариантах функции $g$ задача enu $\left([f]_{0}\right)$ \#Р-полна. Лемма доказана.

Лемма 3. $E c \bumpeq u f\left(x_{1}, \ldots, x_{k}\right) \notin A$, то задача еnu $\left([f]_{\mathrm{C}}\right)$ \#Р-полна.

Доказательство. Поскольку $f$ - не мультиаффинная функция, то найдутся три вектора $\alpha, \beta, \gamma$ такие, что $f(\alpha)=f(\beta)=f(\gamma)=1, f(\alpha \oplus \beta \oplus \gamma)=0$. Без ограничения общности можно считать, что векторы $\alpha, \beta, \gamma$ имеют вид

$$
\begin{aligned}
\alpha & =(0, \ldots, 0,0, \ldots, 0,0, \ldots, 0,0, \ldots, 0,1, \ldots, 1,1, \ldots, 1,1, \ldots, 1,1, \ldots, 1), \\
\beta & =(0, \ldots, 0,0, \ldots, 0,1, \ldots, 1,1, \ldots, 1,0, \ldots, 0,0, \ldots, 0,1, \ldots, 1,1, \ldots, 1), \\
\gamma & =(0, \ldots, 0,1, \ldots, 1,0, \ldots, 0,1, \ldots, 1,0, \ldots, 0,1, \ldots, 1,0, \ldots, 0,1, \ldots, 1),
\end{aligned}
$$

где длины восьми частей каждого из векторов совпадают с длинами частей вектора $\gamma$, состоящими сплошь из нулей и единиц, и равны соответственно $r_{1}, \ldots, r_{8}$, $r_{1}+\ldots+r_{8}=k$.

Рассмотрим функцию из набора $\langle f\rangle_{\mathrm{C}}$

$$
h\left(x_{1}, x_{2}, x_{3}, x_{4}, x_{5}, x_{6}\right)=f\left(0, \ldots, 0, x_{1}, \ldots, x_{1}, \ldots, x_{6}, \ldots, x_{6}, 1, \ldots, 1\right),
$$

где аргументы разбиты на восемь частей длин $r_{1}, \ldots, r_{8}$, состоящих сплошь из нулей, $x_{1}, \ldots, x_{6}$ и единиц, соответственно. Очевидно,

$h(0,0,0,1,1,1)=h(0,1,1,0,0,1)=h(1,0,1,0,1,0)=1, \quad h(1,1,0,1,0,0)=0, \quad h \notin A$. 
Предположим, что набор $\langle f\rangle_{\mathrm{C}}$ содержит функцию $f^{\prime}\left(x_{1}, x_{2}\right)=x_{1} \oplus x_{2}$. Определим функцию вида

$$
h_{1}\left(x_{1}, x_{2}, x_{3}\right)=h\left(x_{1}, x_{2}, x_{3}, \bar{x}_{3}, \bar{x}_{2}, \bar{x}_{1}\right) .
$$

Поскольку

$$
h_{1}(0,0,0)=h_{1}(0,1,1)=h_{1}(1,0,1)=1, \quad h_{1}(1,1,0)=0 \text {, }
$$

то $h_{1} \notin A$ и по лемме 2 задача еnu $\left(\left[h_{1}\right]_{0}\right)$ \#Р-полна.

Покажем, что enu $\left(\left[h_{1}\right]_{0}\right) \propto_{\mathrm{T}}$ enu $\left([f]_{\mathrm{C}}\right)$. Пусть система уравнений

$$
h_{1}\left(z_{s_{i 1}}, z_{s_{i 2}}, z_{s_{i 3}}\right)=1, \quad i=1, \ldots, m,
$$

где $s_{i j} \in \mathbf{N}, z_{s_{i j}} \in\left\{0, x_{s_{i j}}\right\}, i=1, \ldots, m, j=1,2,3$, есть некоторая система из класса $\left[h_{1}\right]_{0}$, зависящая от неизвестных $x_{1}, \ldots, x_{n}$. Этой системе сопоставим систему уравнений из класса $[f]_{\mathrm{C}}$

$$
\begin{aligned}
h\left(z_{s_{i 1}}, z_{s_{i 2}}, z_{s_{i 3}}, y_{s_{i 3}}, y_{s_{i 2}}, y_{s_{i 1}}\right) & =1, \quad i=1, \ldots, m, \\
x_{s_{i j}} \oplus x_{s_{i j}+n} & =1, \quad i=1, \ldots, m, \quad j \in J_{i},
\end{aligned}
$$

где

$$
\begin{aligned}
y_{s_{i j}} & = \begin{cases}1, & \text { если } z_{s_{i j}}=0, i=1, \ldots, m, \\
x_{s_{i j}+n}, & \text { если } z_{s_{i j}}=x_{s_{i j}}, j=1,2,3,\end{cases} \\
J_{i} & =\left\{j \mid z_{s_{i j}}=x_{s_{i j}}, j=1,2,3\right\}, \quad i=1, \ldots, m .
\end{aligned}
$$

Нетрудно показать, что указанные системы уравнений имеют одинаковое число решений. Следовательно, если $\langle f\rangle_{\mathrm{C}} \ni\left(x_{1} \oplus x_{2}\right)$, то enu $\left(\left[h_{1}\right]_{0}\right) \propto_{\mathrm{T}} \operatorname{enu}\left([f]_{\mathrm{C}}\right)$, и задача еnu $\left([f]_{\mathrm{C}}\right)$ \#Р-полна.

Перейдем к рассмотрению общего случая. Если $h(0, \ldots, 0)=1$, то по лемме 2 задача еnu $\left([h]_{0}\right)$ \#Р-полна, откуда вытекает \#Р-полнота задачи еnu $\left([f]_{\mathrm{C}}\right)$. $\mathrm{B}$ дальнейшем считаем $h(0, \ldots, 0)=0$. Рассмотрим возможные варианты функции $h$.

Если $h(0,0,0,0,0,1)=0$, то для функции $h_{2}\left(x_{1}, x_{2}\right)=h\left(0, x_{1}, x_{1}, x_{2}, x_{2}, 1\right)$ либо $h_{2}\left(x_{1}, x_{2}\right)=x_{1} \oplus x_{2}$, либо $h_{2}\left(x_{1}, x_{2}\right)=x_{1} \vee x_{2}$. По доказанному ранее, в любом из этих случаев задача enu $\left([f]_{\mathrm{C}}\right)$ \#Р-полна. Далее считаем $h(0,0,0,0,0,1)=1$.

Если $h(0,0,0,0,1,0)=0$, то для функции $h_{3}\left(x_{1}, x_{2}\right)=h\left(x_{1}, 0, x_{1}, x_{2}, 1, x_{2}\right)$ либо $h_{3}\left(x_{1}, x_{2}\right)=x_{1} \oplus x_{2}$, либо $h_{3}\left(x_{1}, x_{2}\right)=x_{1} \vee x_{2}$. В любом из случаев задача enu $\left([f]_{\mathrm{C}}\right)$ \#Р-полна. Далее считаем $h(0,0,0,0,1,0)=1$.

Функция $h_{4}\left(x_{1}, x_{2}\right)=h\left(0,0,0,0, x_{1}, x_{2}\right)$ принимает одно из двух значений $h_{4}\left(x_{1}, x_{2}\right)=x_{1} \oplus x_{2}$ или $h_{4}\left(x_{1}, x_{2}\right)=x_{1} \vee x_{2}$. При этом задача еnu $\left([f]_{\mathrm{C}}\right)$ \#Р-полна.

Таким образом, при любых вариантах функции $h$ задача enu $\left([f]_{\mathrm{C}}\right)$ \#P-полна. Лемма доказана.

Перейдем к рассмотрению сложности задач определения числа решений систем класса $[f]_{\mathrm{NC}}$. Обозначим

$$
\begin{aligned}
u_{1}\left(x_{1}, x_{2}, x_{3}\right) & =\bar{x}_{1} \bar{x}_{2} \bar{x}_{3} \vee \bar{x}_{1} \bar{x}_{2} x_{3} \vee \bar{x}_{1} x_{2} \bar{x}_{3} \vee x_{1} \bar{x}_{2} x_{3} \vee x_{1} x_{2} \bar{x}_{3} \vee x_{1} x_{2} x_{3}, \\
u_{2}\left(x_{1}, x_{2}, x_{3}, x_{4}\right) & =\bar{x}_{1} \bar{x}_{2} \bar{x}_{3} \bar{x}_{4} \vee \bar{x}_{1} \bar{x}_{2} x_{3} x_{4} \vee \bar{x}_{1} x_{2} \bar{x}_{3} x_{4} \vee x_{1} \bar{x}_{2} x_{3} \bar{x}_{4} \vee x_{1} x_{2} \bar{x}_{3} \bar{x}_{4} \vee x_{1} x_{2} x_{3} x_{4} .
\end{aligned}
$$


Лемма 4. Задачи enu([ $\left.\left.u_{1}\right]_{\mathrm{NC}}\right) u$ enu($\left(\left[u_{2}\right]_{\mathrm{NC}}\right)$ \#P-полньь.

Доказательство. Для наглядности в таблице приведены векторы, на которых соответственно функции $u_{1}$ и $u_{2}$ принимают значение 1 :

\begin{tabular}{|c|c|c|c|c|c|c|}
\hline$x_{1}$ & $x_{2}$ & $x_{3}$ & $x_{1}$ & $x_{2}$ & $x_{3}$ & $x_{4}$ \\
\hline 0 & 0 & 0 & 0 & 0 & 0 & 0 \\
\hline 0 & 0 & 1 & 0 & 0 & 1 & 1 \\
\hline 0 & 1 & 0 & 0 & 1 & 0 & 1 \\
\hline 1 & 0 & 1 & 1 & 0 & 1 & 0 \\
\hline 1 & 1 & 0 & 1 & 1 & 0 & 0 \\
\hline 1 & 1 & 1 & 1 & 1 & 1 & 1 \\
\hline
\end{tabular}

Поскольку $u_{1} \notin A, u_{2} \notin A$, по лемме 3 задачи еnu $\left(\left[u_{1}\right]_{\mathrm{C}}\right)$ и еnu $\left(\left[u_{2}\right]_{\mathrm{C}}\right)$ \#Р-полны, и для доказательства леммы достаточно показать, что

$$
\operatorname{enu}\left(\left[u_{1}\right]_{\mathrm{C}}\right) \propto_{\mathrm{T}} \operatorname{enu}\left(\left[u_{1}\right]_{\mathrm{NC}}\right), \quad \operatorname{enu}\left(\left[u_{2}\right]_{\mathrm{C}}\right) \propto_{\mathrm{T}} \operatorname{enu}\left(\left[u_{2}\right]_{\mathrm{NC}}\right) .
$$

Пусть система уравнений

$$
u_{1}\left(y_{s_{i 1}}, y_{s_{i 2}}, y_{s_{i 3}}\right)=1, \quad i=1, \ldots, m,
$$

где $s_{i j} \in \mathbf{N}, y_{s_{i j}} \in\left\{0,1, x_{s_{i j}}\right\}, i=1, \ldots, m, j=1,2,3$, есть некоторая система из класса $\left[u_{1}\right]_{\mathrm{C}}$, зависящая от неизвестных $x_{1}, \ldots, x_{n}$. Системе (13) сопоставим систему из класса $\left[u_{1}\right]_{\mathrm{NC}}$

$$
\begin{aligned}
u_{1}\left(x_{s_{11}}^{\prime}, x_{s_{i 2}}^{\prime}, x_{s_{i 3}}^{\prime}\right)=1, & i=1, \ldots, m, \\
u_{1}\left(x_{j}, x_{1}, x_{2}\right) & =1, \quad j=3, \ldots, n+2,
\end{aligned}
$$

где

$$
x_{s_{i j}}^{\prime}=\left\{\begin{array}{lll}
x_{1}, & \text { если } y_{s_{i j}}=0, & i=1, \ldots, m, \\
x_{2}, & \text { если } y_{s_{i j}}=1, & j=1,2,3 . \\
x_{s_{i j}+2}, & \text { если } y_{s_{i j}}=x_{s_{i j}}, &
\end{array}\right.
$$

Через $q_{1}, t_{1}$ обозначим соответственно число решений систем (13), (14).

Легко видеть, что если вектор $\left(\alpha_{1}, \ldots, \alpha_{n}\right)$ - решение системы (13), то векторы $\left(0,1, \alpha_{1}, \ldots, \alpha_{n}\right)$ и $\left(1,0, \bar{\alpha}_{1}, \ldots, \bar{\alpha}_{n}\right)$ являются решениями системы $(14)$. Кроме того, векторы $0_{n+2}$ и $1_{n+2}$ есть решения системы (14). Поэтому

$$
t_{1} \geqslant 2 q_{1}+2 \text {. }
$$

Система (14) обладает тем свойством, что, если в ее решении $\left(\beta_{1}, \ldots, \beta_{n+2}\right)$ первые две координаты равны между собой, то $\beta_{1}=\beta_{2}=\ldots=\beta_{n+2}$. Через $t$ обозначим множество решений системы (14), первые две координаты которых равны, соответственно, 0 и 1 . Просто убедиться в том, что

$$
q_{1} \geqslant t_{1}^{\prime}=\left(t_{1}-2\right) / 2 .
$$

Объединяя соотношения (15) и (16), находим, что $q_{1}=\left(t_{1}-2\right) / 2$. Следовательно, $\operatorname{enu}\left(\left[u_{1}\right]_{\mathrm{C}}\right) \propto_{\mathrm{T}} \operatorname{enu}\left(\left[u_{1}\right]_{\mathrm{NC}}\right)$, и задача enu $\left(\left[u_{1}\right]_{\mathrm{NC}}\right)$ \#Р-полна. 
Пусть система

$$
u_{2}\left(y_{s_{i 1}}, y_{s_{i 2}}, y_{s_{i 3}}, y_{s_{i 4}}\right)=1, \quad i=1, \ldots, m
$$

где $s_{i j} \in \mathbf{N}, y_{s_{i j}} \in\left\{0,1, x_{s_{i j}}\right\}, i=1, \ldots, m, j=1, \ldots, k$, есть некоторая система из класса $\left[u_{2}\right]_{\mathrm{C}}$, зависящая от неизвестных $x_{1}, \ldots, x_{n}$. Системе (17) сопоставим систему из класса $\left[u_{2}\right]_{\mathrm{NC}}$

$$
\begin{aligned}
u_{2}\left(x_{s_{i 1}}^{\prime \prime}, x_{s_{i 2}}^{\prime \prime}, x_{s_{i 3}}^{\prime \prime}, x_{s_{i 4}}^{\prime \prime}\right)=1, & i=1, \ldots, m \\
u_{2}\left(x_{1}, x_{2 j-1}, x_{2 j}, x_{2}\right)=1, & j=2, \ldots, n+1
\end{aligned}
$$

где

$$
x_{s_{i j}}^{\prime \prime}=\left\{\begin{array}{lll}
x_{1}, & \text { если } y_{s_{i j}}=0, & i=1, \ldots, m, \\
x_{2}, & \text { если } y_{s_{i j}}=1, & j=2, \ldots, n+1 . \\
x_{2 s_{i j}-1}, & \text { если } y_{s_{i j}}=x_{s_{i j}}, &
\end{array}\right.
$$

Число решений систем (17), (18) обозначим соответственно $q_{2}, t_{2}$. Заметим, что если вектор $\left(\beta_{1}, \ldots, \beta_{2 n+2}\right)$ есть решение системы (18) и $\beta_{1}=\beta_{2}$, то $\beta_{1}=$ $\beta_{2}=\beta_{3}=\ldots=\beta_{2 n+2}$, а в случае $\beta_{1} \neq \beta_{2}$ выполняются неравенства $\beta_{2 j-1} \neq$ $\beta_{2 j}, j=2, \ldots, n+1$. Кроме того, системы (17) и (18) обладают следующими свойствами: если вектор $\left(\alpha_{1}, \ldots, \alpha_{n}\right)$ - решение системы (17), то векторы $\left(0,1, \alpha_{1}, \bar{\alpha}_{1}, \ldots, \alpha_{n}, \bar{\alpha}_{n}\right)$ и $\left(1,0, \bar{\alpha}_{1}, \alpha_{1}, \ldots, \bar{\alpha}_{n}, \alpha_{n}\right)$ являются решениями системы (18); если вектор $\left(0,1, \gamma_{1}, \gamma_{2}, \ldots, \gamma_{2 n}\right)$ есть решение системы (18), то вектор $\left(\gamma_{1}, \gamma_{3}, \gamma_{5}, \ldots, \gamma_{2 n-1}\right)$ есть решение системы $(17)$.

Используя эти свойства и то обстоятельство, что решениями системы (18) является векторы $0_{2 n+2}, 1_{2 n+2}$, находим, что $q_{2}=\left(t_{2}-2\right) / 2$.

Следовательно, enu $\left(\left[u_{2}\right]_{\mathrm{C}}\right) \propto_{\mathrm{T}} \mathrm{enu}\left(\left[u_{2}\right]_{\mathrm{NC}}\right)$, и задача enu $\left(\left[u_{2}\right]_{\mathrm{NC}}\right)$ \#P-полна.

Лемма 5. Если $f\left(x_{1}, \ldots, x_{k}\right) \notin A$, то задача еnu $\left([f]_{\mathrm{NC}}\right)$ \#P-полна.

Доказательство. Рассмотрим возможные варианты значений функции $f$ на векторах $0_{k}$ и $1_{k}$.

Пусть $f\left(0_{k}\right)=f\left(1_{k}\right)=0$. Предположим, что найдется вектор $\alpha \in B_{k}$, для которого $f(\alpha)=1, f\left(\alpha \oplus 1_{k}\right)=0$. Без ограничения общности можем считать

$$
\alpha=(0, \ldots, 0,1, \ldots, 1),
$$

где число нулей $r \in\{1, \ldots, k-1\}$.

Рассмотрим некоторую систему уравнений из класса $[f]_{\mathrm{C}}$

$$
f\left(y_{s_{i 1}}, \ldots, y_{s_{i k}}\right)=1, \quad i=1, \ldots, m
$$

где $s_{i j} \in \mathbf{N}, y_{s_{i j}} \in\left\{0,1, x_{s_{i j}}\right\}, i=1, \ldots, m, j=1, \ldots, k$, зависящую от неизвестных $x_{1}, \ldots, x_{n}$. Системе $(20)$ сопоставим систему из класса $[f]_{\mathrm{NC}}$

$$
\begin{aligned}
f\left(x_{s_{i 1}}^{\prime}, \ldots, x_{s_{i k}}^{\prime}\right) & =1, \quad i=1, \ldots, m, \\
f\left(x_{1}, \ldots, x_{1}, x_{2}, \ldots, x_{2}\right) & =1
\end{aligned}
$$


где число переменных $x_{1}$ в последнем уравнении равно $r$,

$$
x_{s_{i j}}^{\prime}=\left\{\begin{array}{lll}
x_{1}, & \text { если } y_{s_{i j}}=0, & i=1, \ldots, m, \\
x_{2}, & \text { если } y_{s_{i j}}=1, & j=1, \ldots, k . \\
x_{2 s_{i j}-1}, & \text { если } y_{s_{i j}}=x_{s_{i j}}, &
\end{array}\right.
$$

Поскольку из уравнения $f\left(x_{1}, \ldots, x_{1}, x_{2}, \ldots, x_{2}\right)=1$ следует, что $x_{1}=0, x_{2}=1$, то число решений систем (20) и (21) совпадает. Следовательно, справедлива сводимость епu $\left([f]_{\mathrm{C}}\right) \propto_{\mathrm{T}}$ enu $\left([f]_{\mathrm{NC}}\right)$ и, используя лемму 3 , находим, что задача еnu $\left([f]_{\mathrm{NC}}\right)$ \#Р-полна.

Предположим теперь, что $f(\beta)=f\left(\beta \oplus 1_{k}\right)$ для любого вектора $\beta \in B_{k}$. Так как $f \notin A$, то $f \not \equiv 0$ и найдется вектор $\alpha$, для которого $f(\alpha)=1$. Без ограничения общности считаем, что вектор $\alpha$ имеет вид (19). Системе (20) сопоставим систему (21). Легко видеть, что число решений системы (21) равно удвоенному числу решений системы $(20)$. Поэтому еnu $\left([f]_{\mathrm{C}}\right) \propto_{\mathrm{T}}$ enu $\left([f]_{\mathrm{NC}}\right)$, и задача enu $\left([f]_{\mathrm{NC}}\right)$ \#Pполна.

Пусть $f\left(0_{k}\right)=1, f\left(1_{k}\right)=0$. В этом случае из уравнения $f\left(x_{1}, \ldots, x_{1}\right)=1$ следует $x_{1}=0$. Неизвестное $x_{1}$ можно использовать в качестве константы 0 . Поэтому еnu $\left([f]_{0}\right) \propto_{\mathrm{T}}$ enu $\left([f]_{\mathrm{NC}}\right)$ и по лемме 2 задача еnu $\left([f]_{\mathrm{NC}}\right)$ \#Р-полна.

Пусть $f\left(0_{k}\right)=0, f\left(1_{k}\right)=1$. Рассмотрим функцию $f^{\prime}\left(x_{1}, \ldots, x_{k}\right)=f\left(\bar{x}_{1}, \ldots, \bar{x}_{k}\right)$. Так как $f^{\prime} \notin A, f^{\prime}(0)=1, f^{\prime}(1)=0$, согласно доказательству данной леммы в предыдущем случае, задача еnu $\left(\left[f^{\prime}\right]_{\mathrm{NC}}\right)$ \#Р-полна. Очевидно, что справедлива сводимость епu $\left(\left[f^{\prime}\right]_{\mathrm{NC}}\right) \propto_{\mathrm{T}}$ enu $\left([f]_{\mathrm{NC}}\right)$, следовательно, \#Р-полна и задача еnu $\left([f]_{\mathrm{NC}}\right)$.

Пусть $f\left(0_{k}\right)=f\left(1_{k}\right)=1$. Пусть существует вектор $\alpha \in B_{k}$ такой, что $f(\alpha)=1, f\left(\alpha \oplus 1_{k}\right)=0$. Можем считать, что вектор $\alpha$ имеет вид (19). Тогда $f\left(x_{1}, \ldots, x_{1}, x_{2}, \ldots, x_{2}\right)=\bar{x}_{1} \vee x_{2}$, и задача enu $\left([f]_{\mathrm{NC}}\right)$ \#Р-полна.

Предположим теперь, что $f(\alpha)=f\left(\alpha \oplus 1_{k}\right)$ для любого вектора $\alpha \in B_{k}$. Поскольку $f \notin A, f(0)=1$, то найдутся векторы $\beta, \gamma \in B_{k}$ такие, что $f(\beta)=f(\gamma)=1$, $f(\beta \oplus \gamma)=0$. Без ограничения общности считаем, что

$$
\begin{aligned}
& \beta=(0, \ldots, 0,0, \ldots, 0,1, \ldots, 1,1, \ldots, 1), \\
& \gamma=(0, \ldots, 0,1, \ldots, 1,0, \ldots, 0,1, \ldots, 1),
\end{aligned}
$$

где оба вектора разбиты на четыре части длин $r_{1}, \ldots, r_{4}$, сплошь состоящие из нулей и единиц. Рассмотрим функцию

$$
u\left(x_{1}, x_{2}, x_{3}, x_{4}\right)=f\left(x_{1}, \ldots, x_{1}, x_{2}, \ldots, x_{2}, x_{3}, \ldots, x_{3}, x_{4}, \ldots, x_{4}\right),
$$

где аргументы $f$ состоят из $r_{1}, \ldots, r_{4}$ переменных $x_{1}, \ldots, x_{4}$ соответственно. Для функции $u$

$$
\begin{gathered}
u(0,0,0,0)=u(0,0,1,1)=u(0,1,0,1)=1, \quad u(0,1,1,0)=0, \\
u(\lambda)=u\left(\lambda \oplus 1_{4}\right), \quad \lambda \in B_{4} .
\end{gathered}
$$

Если $u(0,0,0,1) \neq u(0,0,1,0)$, то либо $u\left(x_{2}, x_{2}, x_{1}, x_{3}\right)=u_{1}\left(x_{1}, x_{2}, x_{3}\right)$ (определение функции $u_{1}$ дано перед леммой 4$)$, либо $u\left(x_{3}, x_{3}, x_{2}, x_{1}\right)=u_{1}\left(x_{1}, x_{2}, x_{3}\right)$. В этих случаях по лемме 4 задача enu $\left([f]_{\mathrm{NC}}\right)$ \#Р-полна. Аналогично показывается, что при $u(0,1,0,0) \neq u(0,0,0,1)$ или $u(1,0,0,0) \neq u(0,0,1,0)$ задача enu $\left([f]_{\mathrm{NC}}\right)$ \#Pполна. Далее полагаем, что $u(0,0,0,1)=u(0,0,1,0)=u(0,1,0,0)=u(1,0,0,0)$. 
При этом возможны два варианта для $u$, либо $u(0,0,0,1)=0$, либо $u(0,0,0,1)=1$. В первом случае $u\left(x_{1}, x_{2}, x_{3}, x_{4}\right)=u_{2}\left(x_{1}, x_{2}, x_{3}, x_{4}\right)$ (определение функции $u_{2}$ дано перед леммой 4) и по лемме 4 задача еnu $\left([f]_{\mathrm{NC}}\right)$ \#Р-полна. Если $u(0,0,0,1)=1$, то $u\left(x_{2}, x_{1}, x_{1}, x_{3}\right)=u_{1}\left(x_{1}, x_{2}, x_{3}\right)$, и по лемме 4 задача еnu $\left([f]_{\mathrm{NC}}\right)$ \#Р-полна.

Таким образом, для любой не мультиаффинной функции $f$ задача $\mathrm{enu}\left([f]_{\mathrm{NC}}\right)$ \#Р-полна. Лемма и теорема разделимости для задач вида еnu $\left([F]_{\mathrm{NC}}\right)$ доказаны.

\section{Список литературы}

1. Rudeanu S. Boolean Functions and Equations. Elsevier, Amsterdam, 1974.

2. Закревский А. Д. Логический синтез каскадных схем. Наука, Москва, 1981.

3. Гэри М., Джонсон Д. Вычислительные машины и труднорешаемые задачи. Мир, Москва, 1982.

4. Schaefer T. The complexity of satisfiability problems. In: Proc. 10th Annual ACM Symp. Theory of Computing: San Diego, California, May 1-3, 1978.

5. Горшков С. П. О сложности нахождения числа выполняющих наборов значений переменных в задаче "обобщенная выполнимость". В кн.: Тезисы докладов Девятой Всесоюзной конференчии по математической логике. Наука, Ленинград, 1988.

6. Стокмейер Л. Классификация вычислительной сложности проблем. Киберн. сб. (1989) 26, 20-83.

7. Valiant L. The complexity of computing the permanent. Theoretical Computer Sci. (1978) 8, 189-201.

8. Simod J. On the difference between one and many. Lect. Notes in Computer Sci. (1977) 52, 480-491.

9. Valiant L. The complexity of enumeration and reliability problems. SIAM J. Comput. (1979) 8, 410-421.

10. Ахо А., Хопкрофт Д., Ульман Д. Построение и анализ вычислительных алгоритмов. Мир, Москва, 1979.

Статья поступила 09.09.93. 